\title{
Fettleibigkeit erfordert keine Dosisanpassung bei DOAKs bei VTE und Vorhofflimmern
}

Martin AC et al. Direct Oral Anticoagulant Concentrations in Obese and High Body Weight Patients: A Cohort Study. Thromb Haemostasis 2021; 121: 224-233 doi:10.1055/s-0040-1715834

Bei venösem Thromboembolismus (VTE) oder zur Schlaganfallprävention bei Vorhofflimmern werden vorzugsweise direkte orale Antikoagulanzien (DOAKs) verordnet. Ihr breites therapeutisches Fenster erlaubt feste Dosierungen ohne die Notwendigkeit routinemäßiger Kontrollen. Es wird allerdings befürchtet, dass Fettleibigkeit die Pharmakokinetik und Pharmakodynamik verändern und DOAK-Konzentrationen verringern könnte. Dieser Frage geht die Studie nach.

In bisherigen Studien waren nur wenige Patient/-innen mit einem Körpergewicht $\geq 120 \mathrm{~kg}$ enthalten. Martin et al. untersuchten den Zusammenhang zwischen Konzentrationen von Faktor-Xa (FXa) -Inhibitoren, Körpergewicht und Nierenfunktion. Diese Werte wurde dann zwischen Patient/-innen mit hohem Körpergewicht und unselektierter Population verglichen.

Die prospektive Observationsstudie führten die Autor/-innen zwischen Oktober 2017 und Oktober 2019 an 2 Zentren im Vereinigten Königreich durch. In diesen wurden 100 konsekutive Patient/-innen im mittleren Alter von 58 (23-78) Jahren mit einem Körpergewicht $\geq 120 \mathrm{~kg}$ in die Studie aufgenommen. Das mediane Körpergewicht betrug $139 \mathrm{~kg}, 84 \%$ wiesen einen Body-Mass-Index (BMI) $\geq 40 \mathrm{~kg} / \mathrm{m}^{2}$ auf, $31 \%$ waren Frauen. Die KreatininClearance variierte zwischen 67 und 474 ml/min. 58 Patient/-innen im mittleren Alter von 61 (43-78) Jahren wiesen Vorhofflimmern auf, 42 im mittleren Alter von 48 (23-75) Jahren eine VTE. Alle erhielten Apixaban 5 mg 2-mal/Tag oder Rivaroxaban $20 \mathrm{mg}$ 1-mal/Tag wegen Vorhofflimmern oder VTE. Die Messungen der Spitzen- und Talkonzentrationen erfolgten mit speziellen chromogenen Assays.

Insgesamt variierten die Spitzen-DOAKKonzentrationen zwischen 44 und 727 ng/ $\mathrm{ml}$, die Talkonzentrationen zwischen 14 und $299 \mathrm{ng} / \mathrm{ml}$. Die Talkonzentrationen von Apixaban und Rivaroxaban unterschieden sich zwischen den Gruppen mit VTE oder Vorhofflimmern nicht. Zwischen den Spitzen- oder Talkonzentrationen der FXaInhibitoren und dem Körpergewicht oder dem BMI und der Kreatinin-Clearance bestand keine lineare Beziehung. Die Talkonzentrationen von Apixaban bei Personen mit Vorhofflimmern und die Spitzenkon- zentrationen von Rivaroxaban bei Personen mit VTE waren niedriger als in der unselektierten Population. Abweichungen von den erwarteten Konzentrationen gab es jedoch nur bei 2 Talkonzentrationen, 109/116 lagen innerhalb des therapeutischen Bereichs.

\section{FAZIT}

Nach ihrer Studie, so die Autor/-innen, erscheint es unwahrscheinlich, dass Patient/-innen mit hohem Körpergewicht oder Fettleibigkeit mit Standarddosierungen von FXa-Inhibitoren wegen VTE oder Vorhofflimmern unterdosiert sind. Nach ihren Ergebnissen seien bei diesen Patient/-innen nicht per se Konzentrationsmessungen und Dosisanpassungen zu rechtfertigen. Sie halten allerdings weitere Studien zur Erforschung des klinischen Outcomes für erforderlich.

Dr. Gabriele Dobler, Berlin 